\title{
Actividad antioxidante de compuestos naturales nitrogenados
}

\author{
Por R. Maestro-Durán y R. Borja-Padilla \\ Instituto de la Grasa y sus Derivados. Avda. Padre García Tejero no 4. 41012-Sevilla. España
}

\section{RESUMEN}

Actividad antioxidante de compuestos naturales nitrogenados.

Continuando el estudio de los antioxidantes naturales, se incluyen en esta tercera parte los compuestos nitrogenados, clasificados en los siguientes grupos:

Aminoácidos y proteínas.

Productos de la reacción coloreada de Maillard.

Fosfolípidos (con las bases colina y etanolamina) y sus productos de calentamiento.

Riboflavina.

Clorofilas y porfirinas.

Acido úrico y sus derivados.

Se discute su comportamiento como inhibidores de la reacción en cadena de los radicales prooxidantes, por reacción irreversible o por secuestro de estos radicales, así como su acción sinergista con antioxidantes primarios.

Finalmente, se citan las patentes registradas en los últimos años para estos tipos de compuestos, sus derivados y sinergistas, en productos alimentarios o en otros usos.

PALABRAS-CLAVE: Antioxidante (actividad) - Compuestos nitrogenados - Información (artículo)

\section{SUMMARY}

Antioxidant activity of the nitrogeneous natural compounds

As a continuation of the study of natural antioxidants, this third part deals with nitrogeneous compounds, classified in the following groups.

Amino acids and proteins.

Products of the Maillard reaction.

Phospholipids (with choline or ethanolamine) and their heating products.

Riboflavin.

Chlorophylls and porphyrins.

Uric acid and derivatives.

Their behaviour as inhibitors of the chain reaction of prooxidant radicals, by irreversible reaction or by sequestration of those radicals, as well as their synergistic action with primary antioxidants is discussed.

Finally, the patents registered during the last years for these kind of compounds, their derivatives and synergits in food and other uses are cited.

KEY-WORDS: Antioxidant (activity) - Information (paper) - Nitrogeneous compounds.

\section{INTRODUCCION}

En este trabajo se recogen los estudios sobre el carácter antioxidante o sinergista con otros antioxidantes de los compuestos nitrogenados. En trabajos anteriores se han estudiado los mecanismos de oxidación producida por la luz y el oxígeno atmosférico y la protección contra ella ejercida por los compuestos fenólicos, a excepción de los tocoferoles (Maestro, 1993a), por las vitaminas E y C y los carotenoides (Maestro, 1993b).

\section{AMINOACIDOS Y PROTEINAS}

Ciertos aminoácidos pueden tener actividad antioxidante primaria, pero por lo general deben su efectividad al sinergismo con otros compuestos. Aunque esta función sinergista se basa fundamentalmente en secuestrar las trazas metálicas que catalizan la oxidación, hay otros mecanismos, como reacción con compuestos prooxidantes o regeneración de antioxidantes que ya habían sido oxidados.

La cinética de la oxidación del aceite de soja al que se había añadido 500 p.p.m. de diversos aminoácidos mostró que la metionina y la cistina se comportan como antioxidantes, mientras que la cisteína favorece la oxidación. La metionina al $1,0 \%$ en peso se mostró antioxidante para el aceite de cártamo calentado ligeramente (Sims, 1977). Sin embargo, el uso de metionina como antioxidante está muy limitado por el desagradable olor a azufre que se produce al calentar el aceite.

Riison (1980) probó dieciséis aminoácidos a una concentración de $1,0 \%$ en peso en emulsión en aceite de cártamo. De ellos, sólo histidina, treonina, lisina y metionina mostraron actividad antioxidante, mientras que los demás eran prooxidantes. También se ha investigado la actividad antioxidante de la histidina, triptófano, glicina, cisteína y alanina sobre ácido linoleico, linoleato de metilo y linolenato de metilo.

El 5-hidroxi-triptófano muestra capacidad antioxidante en microsomas de hígado de rata (Cadenas, 1989). La acetil-cisteína, por el contrario, favorece la peroxidación de los lípidos en hepatocitos aislados de rata (Dore, 1986).

Se ha sugerido (Aruoma, 1988) que la taurina (ácido 2aminoetano-sulfónico), la hipotaurina y sus metabolitos precursores (ácido cisteico, cisteamina y ácido cisteaminsulfínico) pueden actuar como antioxidantes in-vivo. La car- 
nosina ( $\beta$-alanil-L-histidina), la homocarnosina ( $\gamma$-aminobutil-L-histidina) y la anserina ( $\beta$-alanil-L-histidina) también han sido probadas como antioxidantes in vivo por el mismo autor (Aruoma, 1989).

El glutatión (GSH), que es un tripéptido con un grupo tiol, se encuentra en concentraciones altas en muchas células. Reacciona con muchos oxidantes, como el $\mathrm{H}_{2} \mathrm{O}_{2}$ dando la forma oxidada, que es un disulfuro (GSSG). El glutatión, así como algunos aminoácidos (cisteína, S-metilcisteína y alanina) aumentan considerablemente la capacidad antioxidante de las vitaminas $C$ y $E$ en la oxidación inducida del linoleato de metilo (Rousseau, 1988). Se ha registrado una patente para un producto farmacéutico del que forma parte el glutatión y que protege a las células contra el melanoma maligno (Ohlenschlaeger, 1989).

La gliadina, que es una proteína sencilla derivada del gluten de trigo y arroz y que también se encuentra en la clara de huevo, protege de la oxidación a los lípidos del aceite de cártamo y de sardina (Taguchi, 1988).

Extractos en metanol de proteínas de semillas desengrasadas de varias especies oleícolas (algodón, cacahuete y soja) mostraron efectividad para impedir la oxidación de filetes de buey fritos. Ha sido estudiado así mismo el efecto de hidrolizados de proteínas como sinergistas de antioxidantes fenólicos (Bishov, 1975), con objeto de poder rebajar los niveles de estos antioxidantes primarios sin que tenga lugar la oxidación.

La caseína es una mezcla de fosfoproteínas que existe en un $3 \%$ en la leche de vaca y que inhibe la peroxidación, tanto enzimática como no enzimática, de los ácidos grasos poliinsaturados por inclusión reversible de los mismos.

Se han registrado diversas patentes sobre el empleo de derivados de aminoácidos como antioxidantes:

Oligopéptidos de peso molecular 500-1500 obtenidos por hidrólisis de proteínas de soja (Ibuki, 1989). Antioxidantes para alimentos constituidos por derivados de aminoácidos procedentes de cultivos del microorganismo Proteus mirabilis (Kumagai, 1987). Antioxidantes incoloros para alimentos preparados a partir de aminoácidos y azúcares (Tanaka, 1989). Un preparado de clorhidrato de histidina y glucosa protege durante cinco días al ácido linoleico calentado a $45^{\circ} \mathrm{C}$. Derivados de ácidos grasos saturados e insaturados, de $\mathrm{C}_{4}$ a $\mathrm{C}_{32}$ y aminoácido (metionina) se usan como aditivos para cosméticos, productos farmacéuticos y alimentos.

\section{PRODUCTOS DE LA REACCION DE OSCURECIMIENTO DE MAILLARD}

En uno de los primeros trabajos sobre los productos de la reacción de oscurecimiento, se aisló de la mezcla de reacción de aldohexosas y aminas secundarias las aminohexosa-reductonas, y se demostró que estos compuestos tenían actividad antioxidante probándolos con varios aceites vegetales.

Dos estudios prácticos sobre los compuestos de Amadori producidos en una reacción de Maillard se efectuaron con galletas (Lignert, 1980 a) y salchichas (Lignert, 1980 b). En trabajos más recientes sobre los compuestos antioxidantes que se forman por reacción de ácido dehidroascórbico con triptófano (Namiki, 1982 b y a). Se ha comprobado que la mayor efectividad antioxidante era la de los productos de reacción del ácido dehidroascórbico con triptófano: esta mezcla de reacción era más activa que el BHA y el $\alpha$-tocoferol. En el segundo trabajo se describe el aislamiento e identificación de un compuesto antioxidante de la mezcla de reacción entre el ácido dehidroascórbico y el triptófano. La actividad de este compuesto es entre dos y tres veces mayor que la del BHA a igualdad de concentración molar. Tiene también actividad sinergista con el $\alpha$-tocoferol.

Se han realizado también trabajos sobre los productos antioxidantes de la reacción de Maillard entre histidina y glucosa (Lignert, 1983). Los compuestos preparados por reacción entre aldopentosas, aldohexosas y cetohexosas con aminoácidos fueron probados (Farag, 1982) en la oxidación de ácido linoleico. Los formados por alanina-xilosa y prolina-xilosa mostraron una alta eficacia y pueden servir para proteger a los lípidos de la oxidación. Los correspondientes a la cisteína con glucosa y galactosa tienen una actividad menor, y los de cisteína con xilosa, arabinosa y fructosa tienen un ligero carácter prooxidante. No se encontró correlación entre la estructura química de los aminoácidos o los azúcares y el comportamiento antioxidante de los compuestos.

Los productos de la reacción de Maillard entre fructosa y ácido $\gamma$-amino-butírico o arginina aumentan la estabilidad del linoleato de metilo (Uhl, 1990).

También los fosfolípidos, al calentarse, forman compuestos oscurecidos que muestran características antioxidantes para el linoleato de metilo (Husain, 1986b).

\section{FOSFOLIPIDOS}

Los fosfolípidos han sido estudiados como antioxidantes desde hace muchos años. El interés inicial de estos compuestos surgió del hecho de que muchos aceites crudos eran mucho más estables que después de refinados. Hay que señalar, no obstante, que los fosfolípidos actúan más como agentes quelantes o sinergistas que como antioxidantes primarios. La acción antioxidante de las lecitinas fue atribuida primeramente a la capacidad de los fosfátidos para secuestrar metales, pero en estos datos de la literatura no se hacía distinción entre antioxidantes y sinergistas.

Los fosfátidos de varias semillas oleaginosas (algodón, soja, girasol) fueron probados con éxito como antioxidantes en ghee (mantequilla de leche de búfala) por Bhatia (1978). La capacidad de los fosfolípidos para aumentar la efectividad de los antioxidantes primarios parece variar según el tipo de aceite estudiado (Hudson, 1984 b). El comportamiento sinergista de los distintos fosfolípidos depende también del antioxidante primario considerado. La fosfatidil-etanolamina es el mejor sinergista con los tocoferoles (Dziezig, 1984) y con antioxidantes sintéticos (Hudson, 1984a). Se consume en lugar del antioxidante primario (galato de propilo en este estudio) durante el periodo de 
inducción (Dziezic, 1986). Se han descrito las propiedades antioxidantes de los fosfolípidos naturales y sintéticos. Los resultados indican que la fosfatidil-etanolamina, la fosfatidil-colina, el fosfatidil-inositol y algunas preparaciones comerciales no son efectivas como antioxidantes en aceite de pescado (menhaden). Si se añade una mezcla de fosfatidil-colina y fosfatidil-etanolamina a etoxiquina (1,2-dihidro6-etoxi-2,2,4-trimetil-quinolina), la mezcla resulta un sinergista eficaz, mientras que el fosfatidil-inositol muestra escasa eficacia. La conclusión es que los fosfolípidos tienen poca efectividad como antioxidantes primarios, y que gran parte de su acción sinergista es debida a la parte nitrogenada de la molécula. El sinergismo de los fosfolípidos ha sido estudiado en aceites vegetales por Hudson (1981). El aceite de cacahuete crudo es mucho más estable que el desgomado. Si al refinado se le añaden las lecitinas separadas en la fase de desgomado, el aceite recupera su estabilidad (Gopalakrishna, 1985). La adición de lecitinas de aceite de soja crudas o de sus fracciones soluble e insoluble en etanol aumenta la estabilidad del aceite de girasol y de la manteca. La fracción más activa es la soluble en alcohol, que es también la más rica en fosfatidil-colina y fosfatidil-etanolamina (Nasner, 1986). Los fosfolípidos de yema de huevo, así como la fosfatidil-colina y la fosfatidil-etanolamina, evitan la oxidación del linoleato de metilo calentado a $180^{\circ} \mathrm{C}$, inhibiendo la formación de hidroperóxidos en la fase inicial de la oxidación, mientras que dipalmitil-fosfatidil-colina y dipalmitil-etanolamina sintéticas no tienen capacidad antioxidativa ni sinergismo con el tocoferol (Husain, 1986 a).

El aceite de avena añadido a manteca, sebo o aceite de soja aumenta la estabilidad, medida por el método Ranzimat. La estabilidad de filetes de buey esterilizados en autoclave $110^{\circ} \mathrm{C}$ durante 1 hora aumenta extraordinariamente, debido por una parte a la formación de compuestos de Maillard por parte de los fosfolípidos de las membranas celulares y por otra a la destrucción de la hemoglobina, que cataliza la autooxidación de los lípidos.

Existen las siguientes patentes para el empleo de fosfolípidos como antioxidantes: Acido fosfatídico o sus sales en presencia de $\alpha$-tocoferol (Hudson, 1984 a). Aumento del periodo de inducción del aceite de girasol de 21,8 a 47,0 horas. Empleo de concentrados de fosfolípidos extraídos de aceite de colza con etanol para estabilizar aceites vegetales (Sedlacek, 1990). Uso de lecitinas conteniendo $\geq 80 \%$ de sustancias insolubles en acetona, $\geq 10 \%$ de fosfatidil-colina $y \geq 5 \%$ de fosfatidil-etanolamina para proteger aceites con ácidos grasos poliinsaturados empleados como profilácticos y terapéuticos de arteriosclerosis, infartos de miocardio y trombosis (Mizucuchi, 1989).

\section{RIBOFLAVINA}

El tetrabutilato de riboflavina, soluble en grasas, es un aditivo alimentario autorizado en Japón, aunque no en Estados Unidos, como fuente de vitamina $B_{2}$, pero cuando se absorbe inhibe la autoxidación de los tejidos. El efecto aumenta con $\alpha$-tocoferol (Miyazawa, 1983). Los efectos in vitro han sido estudiados por Aoyama (1985) en manteca y aceite de palma, y Totani (1975) en aceite de pescado. Su poder antioxidante en emulsión ha sido estudiado por Toshiyuki (1987).

\section{CLOROFILAS Y PORFIRINAS}

Aunque las clorofilas y las feofitinas promueven la oxidación de los lípidos en presencia de la luz, en la oscuridad son inhibidoras de la misma. La estabilidad del aceite de colza crudo, que contiene proporciones relativamente altas de clorofila, disminuye drásticamente después del proceso de refinado, donde se pierde el pigmento (Pokorny, 1989).

Se ha estudiado el efecto de 12 porfirinas en la peroxidación de lípidos en homogeneizados de hígado de rata (Imai, 1990). Las porfirinas con grupos 6- y 7-carbetoximetil muestran actividad antioxidante.

\section{ACIDO URICO}

El ácido úrico, que está presente en la sangre a una concentración de $5 \mathrm{mg} 100 \mathrm{ml}^{-1}$ es un antioxidante y protector contra los radicales y agentes oxidantes. Además de secuestrador de radicales hidróxilo, hidroperóxidos de lípidos, oxígeno singulete $\left({ }^{1} \mathrm{O}_{2}\right)$ y radicales oxo-hem, tiene un efecto protector de la estabilidad del ácido linoleico y de la integridad de las membranas de los eritrocitos (Kellogg, 1977). Todo esto llevó a Ames (1983) a afirmar que el ácido úrico puede reemplazar algunas (pero no todas) las funciones antioxidantes del ácido ascórbico. Además, la formación de complejos urato- $\mathrm{Fe}^{3+}$ inhibe espectacularmente la oxidación del ascorbato catalizada por dicho catión, así como la peroxidación de los lípidos en liposomas y en microsomas de hígado de rata (Davies, 1986).

Smith y Lawling (1983) han demostrado que tanto el ácido úrico como el 3-N-ribosil-úrico protegen a los ácidos linolénico y araquidónico de la peroxidación inducida por el ión cobre y a los lípidos de las membranas de los eritrocitos. Farr (1986) ha señalado el pronunciado efecto antioxidante del ácido úrico cuando se añade a cereales de desayuno a concentraciones $>1000 \mu \mathrm{g} / \mathrm{g}$. Teniendo en cuenta que el nivel de ácido úrico en el plasma sanguíneo no aumenta por la ingestión de ácido úrico (Wyngaarden, 1976), la idea de emplear ácido úrico como antioxidante de alimentos no parece completamente absurda.

\section{BIBLIOGRAFIA}

Ames, B.N., Cathcart, R., Schwiers, E. y Hochstein, P. (1983).-«Uric acid: an antioxidant defense in human against oxidant -and radical- caused ageing and cancer: a hypotheses".-Carcinog. Mutagens Environ 2, 6066.-C.A. 100, 83196a.

Aoyama, M., Maruyama, T., Kanematsu, H., Niiya, I., Tsukamoto, M., Tokairin, S. y Matsumoto, T. (1985).-«Studies on the improvement of antioxidant effect of tocopherol. VI. Synergistic effect of L-ascorbyl stearate and riboflavin butylate".-Yukagaku 34, 123-127.

Aruoma, O.I., Halliwell, B., Hoey, B.M. y Butler, J. (1988).-«Antioxidant action of taurine, hypotaurine and their metabolic precursors".-Biochem. J. 256, 251-255. 
Aruoma, O.I., Laghton, M.J. y Halliwell, B. (1989).-«Carnosine, homocarnosine and anserine: could they act as antioxidants in vivo?.».-Biochem. J. 264, 863-869.

Beckel, R.W. y Waller, G.R. (1983).-«Antioxidative arginine-xylose Maillard reaction products: conditions for syntheses».-J. Food Sci. 48, 996-997.

Bhatia, I.S., Kaur, N. y Suxhija, P.S. (1978).-«Role of seed phosphatides as antioxidants for ghee (butter fat)".-J. Sci. Food Agric. 29, 747-752.

Bishov, S.J. y Henick, A.S. (1975).-«Antioxidant effect of protein hydrolyzates in freeze-dried model systems. Synergistic action with a series of phenolic antioxidants".-J. Food Sci. 40, 345-348.

Cadenas, E., Simic, M.G. y Sies, H. (1989).-«Antioxidant activity of 5hydroxytriptophan, 5-hydroxyindole and DOPA against microsomal lipid peroxidation and its dependence on vitamin E».-Free Radical Res. Comm. 6, 11-17,-C.A. 111, 19540h.

Davies, K.J.A., Sevanian, A., Muakkassah-Kelly, S.F. y Hochstein, P. (1986).-«Uric acid-iron complexes. A new aspect of the antioxidant functions of uric acid".-Biochem. J. 235, 747-754.

Dziedzic, S.Z. y Hudson, B.J.F. (1948).-«Phosphatidyl-ethanolamine as a synergist for primary antioxidants in edible oils».-J. Am. Oil Chemists'Soc. 61, 1042-1045.

Dziedzic, S.Z., Robinson, J.L. y Hudson, B.J.F. (1986).-«Fate of propyl gallate and diphosphatidylethanolamine in lard during autoxidation at $120^{\circ} \mathrm{C}$ ".-J. Agric. Food Chem. 34, 1027-1029.

Farag, R.S., Ghali, Y. y Rashed, M.M. (1982).-«Linoleic acid oxidation catalized by Amadori compounds in aqueous media".-Can. Inst. Food Sci. Technol. 15, 174-179.

Farr, D+.R., Loeliger, J. y Savoy, M.C. (1986).-«Foods protected by the important biological antioxidant: uric acid».-J. Sci. Food Agric. 37, 804810.

Gopalakrishna, A.G. y Prabhaker, J.V. (1985).-«Antioxidant efficacy of water gums as a function of relative humidity in peanut oil».-J. Am. Oil Chemists'Soc. 62, 1581-1583.

Hudson, B.J.F. (1984 a).-«Antioxidant synergists».-Brit. U.K. Pat. Appl. GB. $2,123,024$

Hudson, B.J.F. y Gavhami, K. (1984 b).-«Phospholipids as antioxidant synergist for tocopherol in the autoxidation of edible oils".-Lebensm.Wiss. Technol. 17, 191-194.

Hudson, B.J.F. y Mahgoub, S.E.O. (1981).-«Synergism between phospholipids and naturally-ocurring antioxidants in leaf lipids".-J. Sci. Food Agric. 32, 208-210.

Husain, S.R., Terao, J. y Matshushita, S. (1986 b).-«Effect of browning reaction products of phospholipids on autoxidation of methyl linoleate".-J. Am. Oil Chemists'Soc. 63, 1457-1460.

Ibuki, F., Iwami, K. y Kimoto, M. (1989).-«Soybean oligopeptides as antioxidants for food preservation».-Jpn. Kokai Tokkyo Koho JP O,126.392. (89.126.392)

Imai, K., Aimoto, T., Sato, M. y Kimura, R. (1990).-«Antioxidative effect of several porphyrins on lipid peroxidation in rat liver».-Chem. Pharm. Bull. 38, 258-260.-C.A. 112, 171762k

Kawashima, K., Itoh, H. y Chibata, I. (1981).-«Antioxidant effect of peptide in combination with sugar on autoxidation of edible oils».-Agr. Biol. Chem. 45, 987-992.

Kellogg, E.W. III y Fridovich, I. (1977).-«Liposome oxidation and erythrocyte lysis by enzimatically generated superoxide and hydrogen peroxide".-J. Biol. Chem. 252, 6721-6728.

Kumagai, H. y Tochikura, T. (1987).-«Amino acid derivatives and their microbial manufacture as food antioxidants».-Jpn. Kokai Tokkyo Koho JP 62 $25,995(8725,995)$.

Li, H.I., Vladimirov, Y.A. y Deev, A.I. (1989).-«Comparative study of the effect of carnosine and other antioxidants on the chemiluminiscence of a monolayer liposome suspension in the presence of iron (+2) ions".-Biofizica 35, 82-85.-C.A. 112, 174210q.

Lignert, H. y Eriksson, C.E. (1980 a).-«Antioxidative Maillard reaction products. I. Products from sugars and free amino acids".-J. Food Process Preserv. 4, 161-172.

Lignert, H. y Eriksson, C.E. (1980 b).-«Antioxidative Maillard reaction products. II. Products from sugars and peptides or protein hydrolisate".-J. Food Process Preserv. 4, 173-181.

Lignert, H., Eriksson, C.E. y Waller, G.R. (1983).-«The Maillard reaction in foods and nutrition" pag. 335-345.-Walker, G.R. y Feather, M.S. A.C.S. Symposium Series no 215.-American Chemical Society. Washington D.C

Maestro, R. y Borja, R. (1993 a).-«Actividad antioxidante de los compuestos fenólicos".-Grasas y Aceites. 44, 101-106.

Maestro, R. y Borja, R. (1993 b).-«Actividad antioxidante de las vitaminas C y E y la provitamina A»,-Grasas y Aceites. 44, 107-111.
Marcuse, R. (1960).-«Antioxidative effect of aminoacids».-Nature 186, 886887.

Marcuse, R. (1962).-«The effect of some amino acids on the oxidation of linoleic acid and its methyl ester».-J. Am. Oil Chemists'Soc. 39, 97-103.

Miyazawa, T., Sato, C. y Kaneda, T. (1983).-«Antioxidative effects of $\alpha$ tocopherol and riboflavin-butylate in rats dosed with methyl hydroperoxide".-Agric. Biol. Chem. 47, 1577-1582.

Mizucuchi, T., Mochizuki, S., Ishii, T. y Yanai, M. (1987).-«Preservation of composition containing polyunsaturated fatty acids and vitamin A».-Jpn. Kokai Tokkyo Koho 01,128.918. (01 128.918).

Namiki, M., Hayashi, T. y Shigeta, A. (1982 a).-«Isolation and identification of an antioxidant product from the reaction mixture of dehydroascorbic acid with tryptophan».-Agric. Biol. Chem. 46, 1207-1212.

Namiki, M., Shigeta, A. y Hayashi, T. (1982 b).-«Antioxidant effect of the reaction mixture of dehydroascorbic acid with tryptophan".-Agr. Biol. Chem. 45, 1199-1206.

Nasner, A. (1985).-«Antioxidizing properties of lecithin».-Lipid Oxid.-Biol. Food Chem. Aspects. 187-196.-Contrib. LIPIDFORUM/SIK Symp. 1985 (Pub. 1986).

Ohlenschlaeger, G. (1989).-«Galenic pharmaceuticals containing tripeptides for the treatment of metabolic disorders of disecided cells".-Ger. Offen DE 3.722.647.

Pokorny, Y., Davidek, J., Holasova, M., Parizkova, C.J. y Cmolin, J. (1989).«Effects of the method of oil extraction, refining and hydrogenation on the content of pigments in rapeseed oil».-Sh. Uvtiz. Potravin Vedg. 7, 89-101.-C.A. 111, 230930b.

Rhee, C. y Kim, D.H. (1975).-«Antioxidant activity of acetone extracts obtained from a caramelization-type browning reaction".-J. Food Sci. 40, 460-462

Riison, T., Sims, R.J. y Fioriti, J.A. (1980).-«Effect of amino acids on the autoxidation of safflower oil in emulsions".-J. Am. Oil Chemists'Soc. 57, 354-359.

Rousseau, C., Richard, C. y Martin, R. (1988).- «Synergy effects of glutathion and amino acids on the antioxidant efficiency of vitamin $\mathrm{C}$ in the induced oxidation of methyl linoleate».-J. Chim. Phys-Chim. 85, 145-146.

Sangor, M.R. y Pratt, D.E. (1974).-Lipid oxidation and fatty acid changes in beef combined with vegetables and textured vegetable protein".-J. Am. Diet. Ass. 64, 268-270.

Sedlacek, J., Ranny, M., Hrdina, P. y Novak, J. (1990).-«Phospholipid concentrated and method for the production".-Czech. CS 259440.

Sims, R.J. y Fioriti, J.A. (1977).-«Methional as an antioxidant for vegetable oils".-J. Am. Chemists'Soc. 54, 4-7.

Sliwiok, J. y Siechowski (1970).-«Kinetic antioxidant properties of aminoacids".-Riv. Ital. Sostanze Grasse 47, 73-75.

Smith, R.C. y Lawing, L. (1983).-«Antioxidant activity of uric acid and 3-Nribosyl-uric acid with unsatured fatty acids and erytrocyte membranes".Arch. Biochem. Biophys. 273, 166-172.

Taguchi, K., Iwami, K., Kawabata, M. y lbuki, F. (1988).-«Antioxidant effects of wheat gliadin and hens egg white in powder model systems: protection against oxidative deteriorization of safflower oil and sardine oil».Agric. Biol. Chem. 52, 539-542.

Tanaka, M., Fujimoto, K. y Hoshi, M. (1989).-«Manufacture of food antioxidants from amino acids and sugars".-Jpn Kokai Tokkyo Koho JP 01 10,971 (89 10,971).

Toshiyuki, T., Yamamoto, A. y Mineshita, T. (1987).-«Antioxidant effect of riboflavin tetrabutylate in emulsion».-J. Food Sci. 52, 1377-1380.

Totani, Y., Totani, N. y Matsuo, N. (1975).-«Effect of riboflavin tetrabutylate on the autoxidation of oils and fats".-J. Japan Soc. Food Nutr. Food Sci. 28, 41-43.

Uhl, J.C. y Eichner, K. (1990).-«Zum Einfluss von Antioxidantien natürlichen Ursprungs auf die Stabilität von Linolsäuremethylester in Modellsystemen".-Fett. Wiss. Technol. 92, 355-361.

Wyngaarden, J.T. y Kelly, W.N. (1976).-«Goot and hyperuricemia»-1-528. Grune and Statten. Nueva York.

Zipprin, Y.A., Rhee, K.S., Carpenter, Z.L., Hostetler, R.L., Terrell, R.N. y Rhee, K.C. (1981).-"Glandless cottonseed, peanut and soy protein ingredients in ground beef patties: Effect on rancidity and other quality factors".-J. Food Sci. 46, 58-61.

(Recibido: Mayo 1992) 